\title{
Análisis de la calidad de energía eléctrica en una subestación de 300 kVA
}

\section{Analysis of the quality of electrical energy in a $300 \mathrm{kVA}$ substation}

\author{
MARTÍNEZ-RODRÍGUEZ, Guillermo Miguel†*, CRUZ-ISIDRO, Berenice de Jesús, GARRIDO- \\ MELENDEZ, Javier y JIMÉNEZ-RIVERA, Jesús
}

Universidad Veracruzana

ID $1^{\text {er }}$ Autor: Guillermo Miguel, Martínez-Rodríguez / ORC ID: 0000-0001-9760-7893, arXiv Author ID: Guillermo\#2, CVU CONACYT ID: 947594.

ID $1^{\mathrm{er}}$ Coautor: Berenice de Jesús, Cruz-Isidro / ORC ID: 0000-0002-8378-2321, CVU CONACYT ID: 1001204

ID $2^{\text {do }}$ Coautor: Javier, Garrido-Melendez / ORC ID: 0000-0001-9143-408X, Researcher ID Thomson: C-9373-2018, CVU CONACYT ID: 739319

ID $3^{\text {er }}$ Coautor: Jesús, Jiménez-Rivera / ORC ID: 0000-0002-3134-6405, Researcher ID Thomson: T-2722-2018, CVU CONACYT ID: 599539

\section{Resumen}

En este estudio se presentan los resultados de las mediciones de los parámetros eléctricos obtenidos por el analizador trifásico de energía eléctrica, se utilizó una metodología basada en el manual propio del equipo analizador, así como de las normas nacionales e internacionales como del instituto IEEE, de la empresa CFE, y de libros sobre el tema, en los que se establecen los límites y requerimientos para una buena calidad de la energía eléctrica necesaria para el usuario del servicio eléctrico. La contribución abarca desde la conexión de entrada del equipo analizador, así como su instalación a la salida en baja tensión en el transformador, verificando en el equipo los diagramas vectoriales y senoidales para corroborar que su conexión sea adecuada, también se consideraron las medidas de seguridad en la instalación del equipo de medición, en todo el documento se mencionan las características y la interpretación de cada parámetro eléctrico analizado.

Calidad de la energía, Desbalance, Parámetros

\begin{abstract}
In this research are presented the results of the measurements of the electrical parameters obtained by the three phase analyzer of electrical energy, there was used a methodology based on the own manual of the analyzer equipment, well as the national and international standards as well the IEEE Institute, the CFE company, and books of the subject, in which are stablished the limits and requirements for a good quality of the electrical energy necessary for the user of the electrical service. The contribution covers from the conection of the entry of the analyzer equipment until the instalation to the exit of low tension in the transformer, verifying in the equipment the vectorial diagrams and senoidals for corroborate that their conection be adequate, also were considered the measurements of the security in the instalation of the measurement equipment, throughout the document are mentioned the characteristics and the interpretation of each electrical parameter analyzed.
\end{abstract}

Power Quality, Unbalance, Parameters

Citación: MARTÍNEZ-RODRÍGUEZ, Guillermo Miguel, CRUZ-ISIDRO, Berenice de Jesús, GARRIDO-MELENDEZ, Javier y JIMÉNEZ-RIVERA, Jesús. Análisis de la calidad de energía eléctrica en una subestación de 300 Kva. Revista de Operaciones Tecnológicas. 2019. 3-11: 12-20

\footnotetext{
* Correspondencia del Autor (Correo electrónico: guillmartinez@uv.mx)

$\dagger$ Investigador contribuyendo como primer autor.
} 


\section{Introducción}

Actualmente se tiene el interés de las empresas suministradoras de energía eléctrica y de los usuarios del servicio eléctrico, del cumplimiento de los parámetros de la energía eléctrica que se establecen en las normas nacionales e internacionales, debido a que cualquier desviación de la magnitud o frecuencia puede ocasionar un disturbio en la operación del servicio eléctrico, por este motivo se instaló un equipo de medición portátil de la calidad de la energía marca Fluke 435 II, para realizar las mediciones eléctricas a la salida en baja tensión de un transformador de $300 \mathrm{kVA}$ y así determinar el comportamiento de los parámetros de energía eléctrica.

En el estudio participan alumnos y docentes, con el fin de tener un conocimiento de las conexiones a realizar en la salida del transformador, tomando en cuenta la seguridad que se debe tener para trabajar en equipos eléctricos energizados, así como interpretar los datos proporcionados el equipo de medición a través del software Power $\log$ y una computadora.

Se describen los valores encontrados durante la medición de parámetros como, desequilibrio de tensión y corriente, frecuencia, fluctuaciones, armónicos, parpadeo de tensión, huecos y tensiones, así como eventos registrados durante la medición.

\section{Descripción}

El equipo analizador es propiedad de la Universidad Veracruzana, se instaló del $11 / 07 / 2019$ al 17/07/2019, en la salida en baja tensión del transformador de $300 \mathrm{kVA}, 13.2$ $\mathrm{kV} / 220-127 \mathrm{~V}$, conexión delta-estrella tipo de enfriamiento ON-AN, que alimenta parte del total de las cargas eléctricas de la Universidad Veracruzana campus Coatzacoalcos, los edificios que se alimentan de este transformador son, laboratorios de Química básica y Química pesada, el edificio "D" Ingeniería Eléctrica, el edificio " $\mathrm{C}$ " Ingeniería Civil, el edificio administrativo directivo de las facultades de Contabilidad, Administración, Enfermería, e ingenierías Civil, Mecánica y Eléctrica.

\section{Antecedentes}

Los edificios mencionados eran alimentados por otro transformador de la misma capacidad, que se encontraba ubicado en otro lugar, tenia una antigüedad de 28 años aproximadamente, en el que se encontraron valores de armónicos en el neutro de $131.06 \%$, valores arriba de lo permitido en las normas.[7]. La Universidad Veracruzana por motivos de modernización, sustituyó este transformador por un transformador nuevo de $300 \mathrm{kVA}$ en el que se realiza el estudio.

\section{Definiciones}

Las fluctuaciones (caídas) son desviaciones rápidas con respecto a la tensión normal, la duración puede variar desde medio ciclo hasta unos segundos como se define en EN61000-430.

Dip o Sag. Una disminución de entre 0.1 y $0.9 \mathrm{pu}$ en rms de voltaje o corriente a la frecuencia de potencia durante periodos de 0,5 ciclos a 1 min. [4]

Swell. Un aumento en el voltaje o la corriente eficaz en la frecuencia de potencia durante duraciones de 0.5 ciclos a 1 minuto. Los valores típicos son de $1.1-1.8$ pu. [4]

Flicker. Impresión de inestabilidad de la sensación visual inducida por un estímulo luminoso cuya luminosidad o la distribución espectral fluctúa con el tiempo. [4].

Factor de cresta FC. Indica la cantidad de distorsión un FC de 1,41 significa que no hay distorsión y superior a 1,8 significa alta distorsión.[1]

EN 50160. Nombre de límites usados para la supervisión de la calidad de la energía eléctrica y detección de eventos [1].

Armónico (componente). Un componente de orden mayor que uno de la serie Fourier de una cantidad periódica, por ejemplo, en sistemas de $60 \mathrm{~Hz}$, el armónico orden $3^{\circ}$, también es conocido como el "tercer armónico", es $180 \mathrm{~Hz} \cdot[8]$. 
Calidad de la energía. Características eléctricas de un punto determinado del sistema eléctrico, evaluadas con respecto a un conjunto de parámetros y sus valores de referencia, relacionados a la compatibilidad electromagnética entre el suministrador y los clientes.[5].

\section{Instalación del equipo y toma de datos}

En la instalación del equipo analizador de energía, se tomaron las recomendaciones del manual del fabricante. El equipo tiene 4 conexiones de entrada BNC (Bayonet NeillConcelman) para pinzas de corriente y 5 entradas de banana para voltajes.[1].

El manual recomienda siempre que sea posible desenergizar los sistemas de energía antes de hacer las conexiones. Usar siempre equipo de protección personal, así como evitar trabajar solo [1], en nuestro caso las conexiones se realizaron entre dos o tres alumnos con la supervisión del docente, se utiliza casco, lentes, guantes de carnaza, botas de seguridad eléctrica.

En un sistema trifásico, para las conexiones de corriente, se colocan las pinzas alrededor de los conductores de fase $\mathrm{A}(\mathrm{L} 1)$, B(L2), C(L3) y neutro, las pinzas están marcadas con una flecha que indica la polaridad de señal correcta, (la flecha debe indicar hacia la carga).

Para las conexiones de voltaje, se empieza con la conexión a tierra y después en sucesión $\mathrm{N}, \mathrm{A}(\mathrm{L} 1), \mathrm{B}(\mathrm{L} 2), \mathrm{C}(\mathrm{L} 3)$. Como se muestra en la figura No. 1.

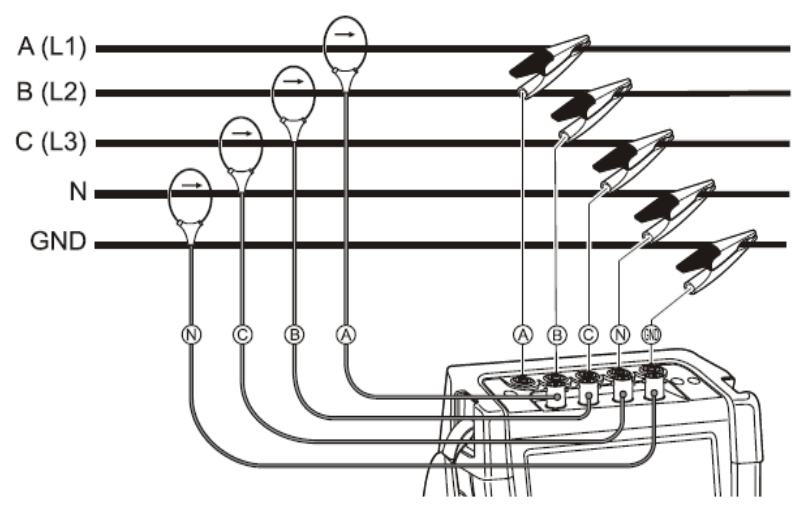

Figura 1 Conexión del analizador al sistema de distribución de conexión trifásico

Fuente: Manual de uso del analizador Fluke 435 II
Antes de realizar las mediciones, se debe configurar el analizador según la tensión de línea, la frecuencia y la configuración del cableado del sistema de alimentación que se desee medir.

Los valores numéricos adicionales corresponden a la tensión y corriente de la fase fundamental, frecuencia y ángulos de fase, en el encabezado de la pantalla muestra los valores rms de tensión, en la figura 2 se muestra la pantalla del fasor para comprobar si los cables de tensión y las pinzas amperimétricas están conectados correctamente las tensiones de fase y las corrientes $\mathrm{A}(\mathrm{L} 1), \mathrm{B}(\mathrm{L} 2), \mathrm{C}(\mathrm{L} 3)$ deben de aparecer en secuencia de sentido de las agujas del reloj. El vector del canal de referencia A(L1) se orienta en la dirección horizontal positiva.

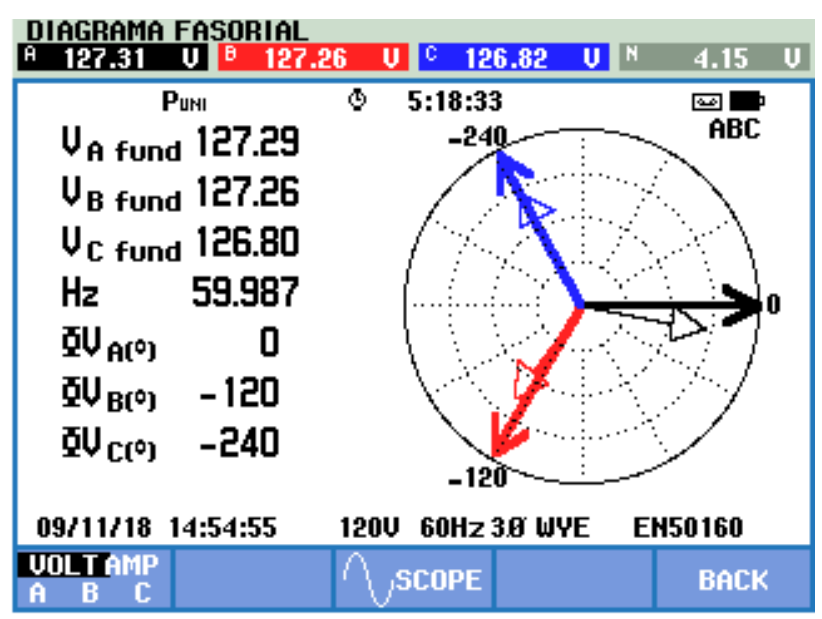

Figura 2 Diagrama de vectores conexión correcta del analizador

Fuente: Elaboración Propia

También se puede ver la pantalla del multímetro donde ofrece un resumen de las variables, por ejemplo, tensiones, corrientes, frecuencia, el factor de cresta FC que indica la cantidad de distorsión, esta pantalla se utiliza para obtener una primera impresión del rendimiento del sistema de alimentación antes de examinar el sistema en detalle, en la figura 3 y 4 se muestran algunos valores. 


\begin{tabular}{|c|c|c|c|c|}
\hline \multicolumn{5}{|c|}{ REGISTRADOR } \\
\hline & PunII & $5: 20$ & & \\
\hline & H & B & C & $\mathrm{NA}$ \\
\hline \multirow{2}{*}{$U_{\text {fund } \lambda}$} & 127.46 & 127.37 & 126.77 & $3.71=$ \\
\hline & 月 & B & C & $\mathrm{N}$ \\
\hline \multirow[t]{2}{*}{ CF U } & 1.41 & 1.42 & 1.41 & 1.59 \\
\hline & A & B & C & $\mathrm{N}$ \\
\hline \multirow[t]{2}{*}{ A pk } & 336.1 & 344.1 & 383.0 & 51.9 \\
\hline & A & B & C & $\mathrm{N}$ \\
\hline Pfund & 224.8 & 218.6 & 245.9 & 3.5 \\
\hline \multicolumn{2}{|l|}{$09 / 11 / 18$} & $120060 \mathrm{H}$ & 38 WYE & EN50160 \\
\hline DODW & & TREFD & EUEHTS & $\begin{array}{l}\text { STOP } \\
\text { START }\end{array}$ \\
\hline
\end{tabular}

Figura 3 Diagrama de vectores conexión correcta del analizador

Fuente: Elaboración Propia

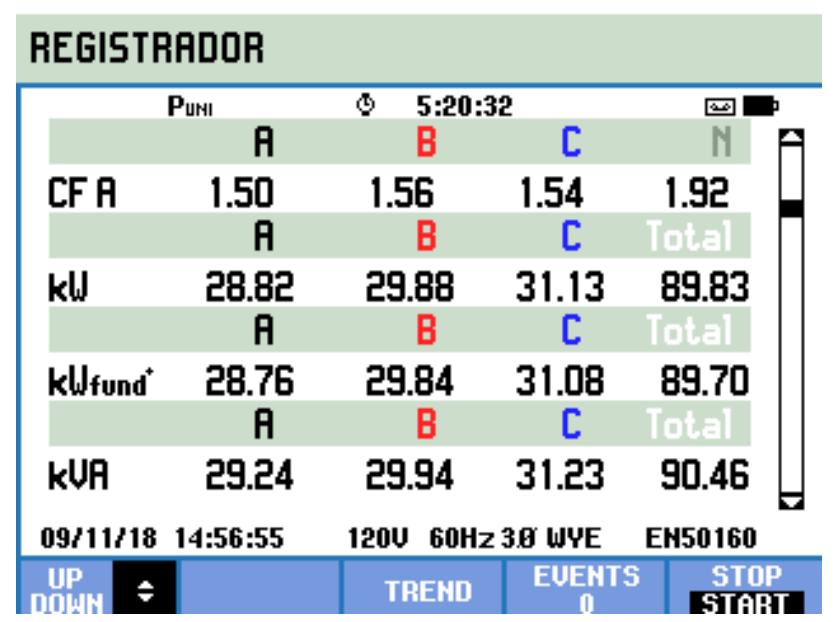

Figura 4 Diagrama de vectores conexión correcta del analizador

Fuente: Elaboración Propia

En la figura 5, muestra la calculadora de pérdidas de energía, que se toma una pantalla con el equipo conectado a la red de baja tensión, con esta impresión el equipo ayuda a determinar donde se producen perdidas de energía, por ejemplo, los $\mathrm{kW}$ efectivos determinan la perdida que se debe al transporte de la potencia efectiva, $62.5 \mathrm{~W}$, pérdidas por $\mathrm{kW}$ reactivos $0.54 \mathrm{~W}$, por desequilibrio y pérdidas por corrientes en el conductor neutro.

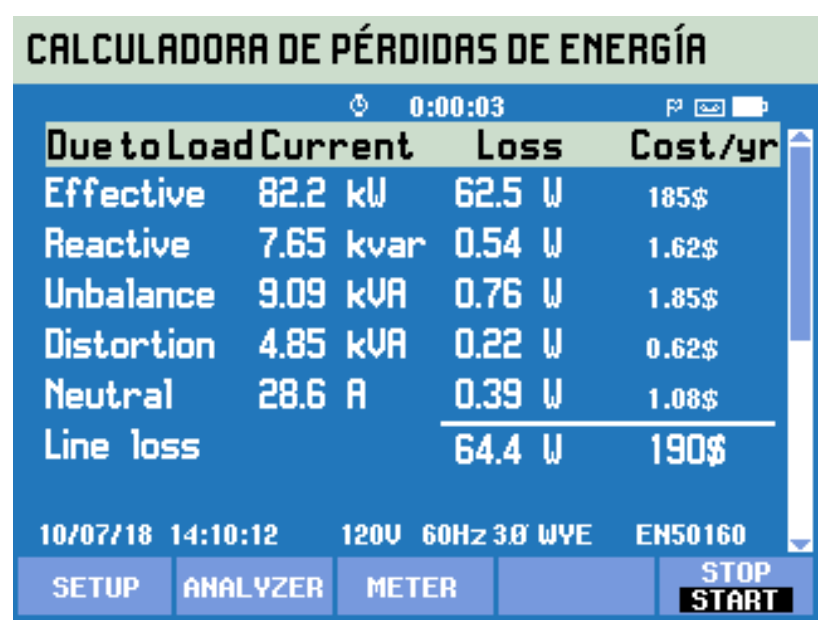

Figura 5 Calculadora de pérdidas de energía Fuente: Elaboración Propia

\section{Análisis de los resultados de las mediciones}

\section{Flicker}

El número de variaciones por minuto, en acometidas de baja, media y alta tensión en estado estacionario, debe limitarse de acuerdo con la tabla 1 de la especificación [3], que se indica a continuación:

Indicador Pst límite $<=1$.

Indicador Plt límite $<=0,65$

En la figura 6 el día 10/07/19 a las 9:55:55 p.m en la fase $\mathrm{BN}$ y $\mathrm{CN}$ respectivamente se muestra un valor de Pst $=2.015$ y Pst $=3.081$, (durante 10 minutos) que sobrepasa el valor indicado en la especificación [3].

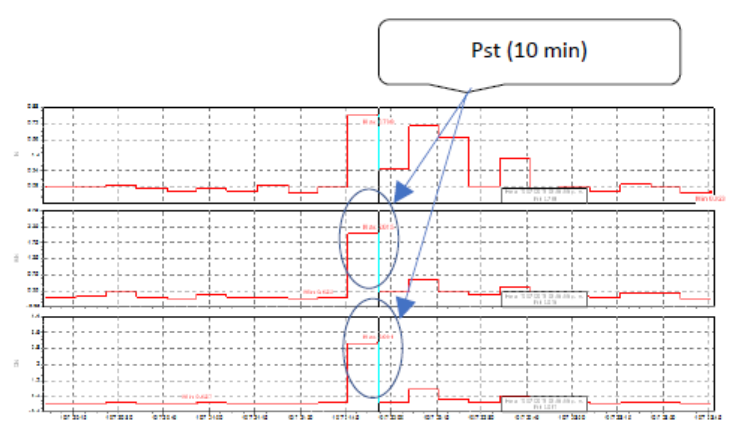

Figura 6 Variación periódica de amplitud de tensión de corto plazo (Pst 10 minutos)

Fuente: Elaboración Propia

En la figura 7 el día 11/07/19 a las 12:45 a.m. en la fase $\mathrm{BN}$ y $\mathrm{CN}$ respectivamente se muestra un valor de Plt $=0.89 \mathrm{y} \mathrm{Plt}=1.354$ en un tiempo (2 horas) sobrepasa el valor indicado en la especificación [3]. 


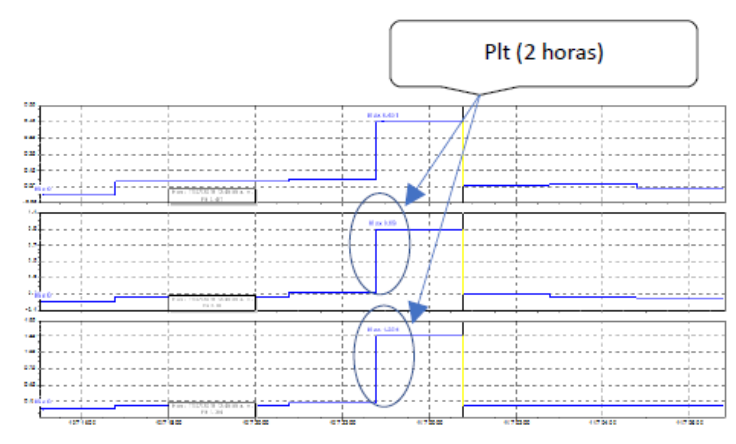

$\square$

Figura 7 Variación periódica de amplitud de tensión de largo plazo (Plt 2 horas)

Fuente: Elaboración Propia

\section{Fluctuaciones}

Durante una caída de tensión, está desciende; durante una subida de tensión, está aumenta. En sistemas trifásicos, se inicia una caída cuando la tensión en una o más fases desciende por debajo del umbral de caída y finaliza cuando todas las fases son iguales o superiores a la histéresis plus del umbral de caída. Las condiciones de activación para las caídas y las subidas son el umbral y la histéresis. [1]

Durante las mediciones se tuvo el registro de un evento No. 9 (DIP) a las 21:50:30 $606 \mathrm{mseg}$, en la figura 8 se observa una caída de tensión en la fase $\mathrm{C}$ respecto a las fases A y B, se detalla que se tuvo un hueco con una duración de 99 ms, o sea 5.94 ciclos, según [4] menciona que el tiempo de duración de un DIP o SAG es de 0.5 a 30 ciclos.

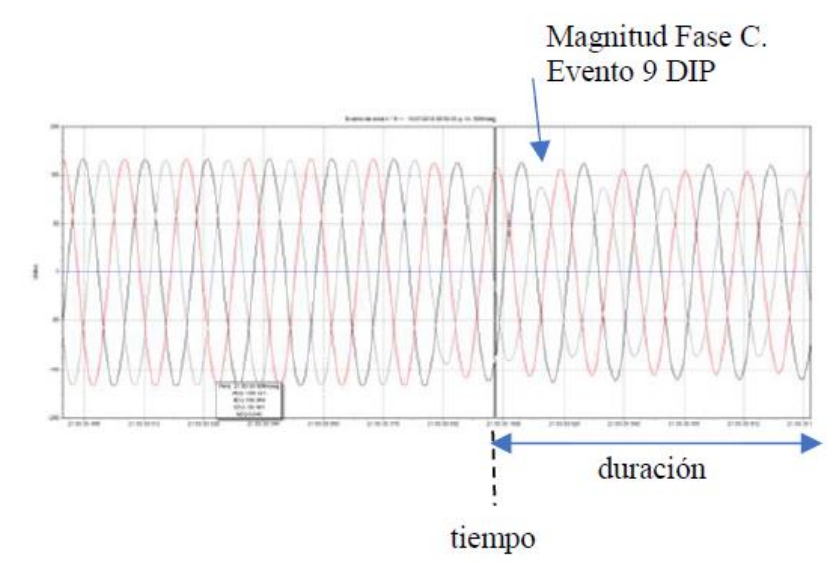

Figura 8 Evento de onda DIP No. 9

Fuente: Elaboración Propia

En la figura 9 se aprecia el detalle del evento No. 9 (DIP) de la caída de tensión, en la fase $\mathrm{C}$ y la variación de la corriente en esta misma fase.

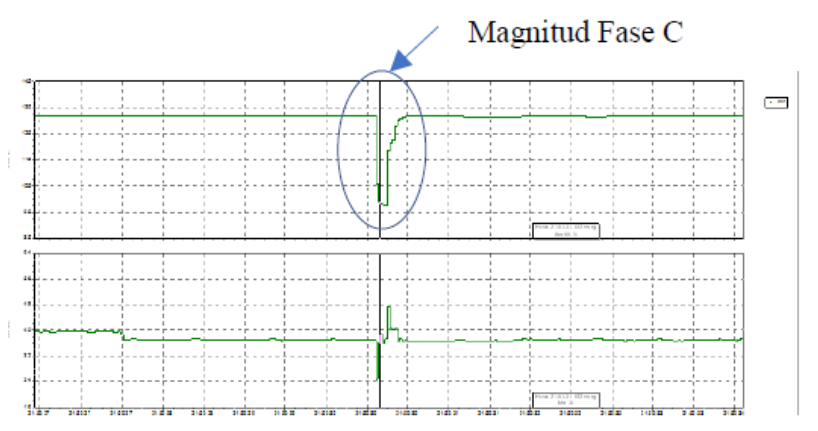

Figura 9 Detalle evento de onda No. 9 (DIP) Fuente: Elaboración Propia

Durante las mediciones se tuvo el registro del evento No. 12 (SWELL) a las 21:50:30 $825 \mathrm{mseg}$. En la figura 10 se observa una subida de tensión en la fase A respecto a las fases B y C, se detalla que se tuvo un incremento con una duración de $24 \mathrm{~m}, 34 \mathrm{~s}, 30 \mathrm{~ms}$, superando lo indicado en [4] que el tiempo de duración de un SWELL es de 0.5 a 30 ciclos.

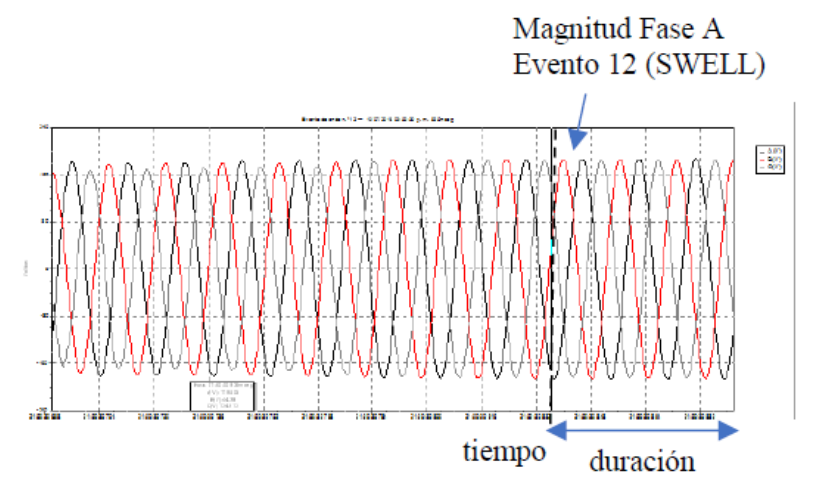

Figura 10 Evento de onda No. 12 (SWELL)

Fuente: Elaboración Propia

\section{Armónicos}

Los armónicos son distorsiones periódicas de las ondas sinusoidales de potencia, corriente o tensión, en la figura 11 los armónicos se mostrarán como porcentaje fundamental con visualización en evolución temporal, que se representan los armónicos a lo largo del tiempo.

Los efectos de los armónicos se deben tanto a la corriente como al voltaje, aunque se producen en la corriente [9].

Los limites de distorsión de Voltaje para tensiones de $V \leq 1.0 \mathrm{kV}$, el armónico individual en $(\%)$ debe ser menor a 5.0, y para la distorsión total de armónico (THD) en (\%) no debe superar el 8.0, según [10] y [11]. 
En la figura 11 se muestra la distorsión de armónica total (THD) por cada fase y neutro, el 10/07/19 al 11/07/19, observando que las fases A, B y C, están en un valor menor de lo indicado en la norma, pero en el caso de la distorsión de armónico total (THD) en el neutro se tienen valores superiores a $8 \%$ en el transcurso de la medición, siendo el más elevado el 10/07/2019 a las 8:55:55 pm, con un valor de $158.32 \%$.

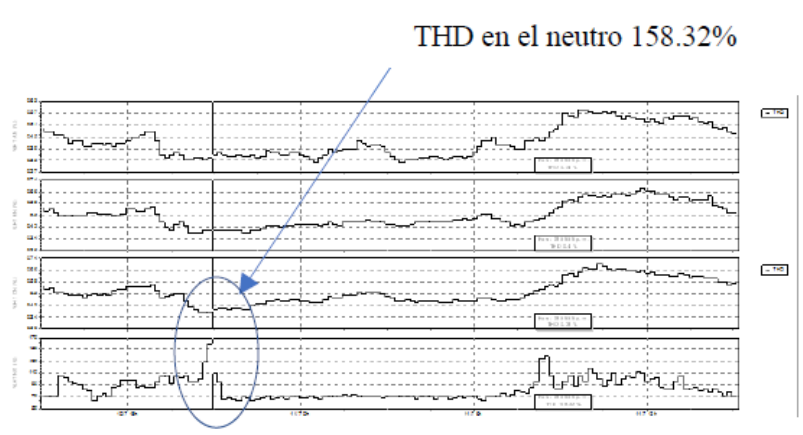

Figura 11 Distorsión armónica fase A, B y C y total Fuente: Elaboración Propia

En la figura 12 se muestra la distorsión de armónica total de corriente (THD) en la opción de histograma, la barra de la fase AN tiene una distorsión armónica total (máximo) de $22.12 \%$, media $12.46 \%$ y mínimo $4.85 \%$, y la distorsión de armónico en el neutro (máximo) tiene $327.67 \%$, medio $170.51 \%$ y mínimo $69.34 \%$,

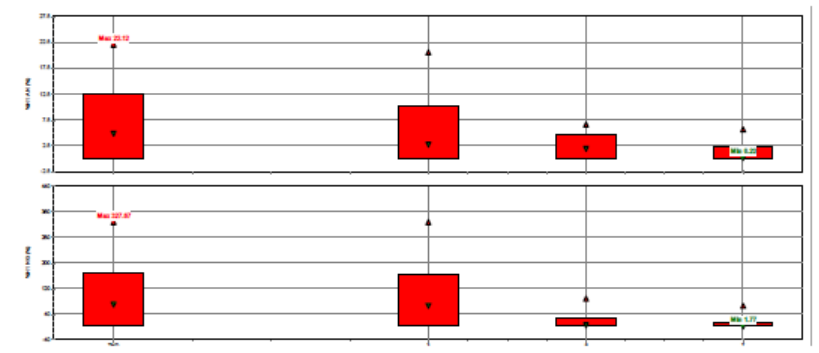

Figura 12 Histograma de distorsión armónica fase AN y Neutro

Fuente: Elaboración Propia

En la norma (IEEE 519-2014) menciona que la Distorsión Armónica Total (THD). Es usada para definir el efecto de los armónicos de voltaje del sistema de potencia; se expresa como un porcentaje el cual es la sumatoria de todos los valores de distorsión armónica de voltaje, la ecuación que la define es: [10], [7].

$T H D=\frac{\sqrt{\sum_{h=2}^{H} V_{h}^{2}}}{V 1} * 100$
Donde:

$\mathrm{V}_{\mathrm{h}}=$ Los voltajes armónicos.

$\mathrm{V}_{1}=$ Voltaje fundamental.

Respecto a la distorsión armónica total de corriente (THD) se tiene: [10], [7].

$T H D=\frac{\sqrt{\sum_{h=2}^{H} I_{h}^{2}}}{I 1} * 100$

Donde:

Ih $=$ Las corrientes armónicas .

I1 $=$ Corriente fundamental.

También se menciona que necesario considerar los parámetros de THD y TDD de la red eléctrica analizada y comparar los datos con las referencias establecidas en las normas. [8], [12]. La Distorsión total de demanda (TDD), Es la relación entre la corriente armónica y la demanda máxima de la corriente de carga y se calcula con la siguiente ecuación 3, [12].

$T D D=\frac{\sqrt{\sum_{h=2}^{H} I_{h}^{2}}}{I_{L}} * 100$

Las normas IEEE Std 519-2014 y la Especificación CFE L0000-45, especifican los mismos límites de distorcion de armónicos de corriente y voltaje, para establecer el porcentaje de componente armónico individual máximo de corriente para armónicas impares (CAIMC), se elige la impedancia relativa $Z_{r}$ es igual a la Corriente de cortocircuito ICC entre la corriente de línea IL.

El valor de la corriente de corto circuito es proporcionado por la compañía suministradora (CFE), en este caso el valor es de 7024 A. [12]. Tomado la corriente de línea de como valor promedio $=234$ amperes, sustituyendo valores da:

$Z_{R}=\frac{7024}{234}=30$

En la tabla 1, que se muestra a continuación se encuentra en que renglón de impedancia relativa corresponde, se observa que para un armónico individual $<11 \mathrm{~h}$ corresponde un valor menor $7 \%$, y para una distorsión de armónica total de demanda, menor a $8 \%$. 


\section{Máxima distorsión de la corriente armónica en} porcentaje de IL

\begin{tabular}{|c|c|c|c|c|c|c|}
\hline \multirow[t]{2}{*}{$\begin{array}{l}\text { Impedancia } \\
\text { relativa } \\
\text { (ICC/IL) }\end{array}$} & \multicolumn{5}{|c|}{$\begin{array}{l}\text { Componente armónico individual máximo } \\
\text { de corriente, para armónicas impares } \\
\text { (CAIMC) } \\
(\%)\end{array}$} & \multirow{2}{*}{$\begin{array}{l}\text { Distorsión } \\
\text { armónica } \\
\text { total de } \\
\text { demanda } \\
\text { (DATD) } \\
\%\end{array}$} \\
\hline & $\begin{array}{l}h \\
<11\end{array}$ & $\begin{array}{l}11 \\
\leq h \\
<17\end{array}$ & $\begin{array}{l}17 \\
\leq h \\
<23\end{array}$ & $\begin{array}{l}23 \\
\leq h \\
<35\end{array}$ & $\begin{array}{l}h \\
\geq 35\end{array}$ & \\
\hline $\begin{array}{l}(\mathrm{Icc} / \\
\mathrm{IL})<20\end{array}$ & 4 & 2 & 1,5 & 0,6 & 0,3 & 5 \\
\hline $\begin{array}{l}20 \leq \\
(\text { Icc } / \\
\text { IL })<50\end{array}$ & 7 & 3,5 & 2,5 & 1 & 0,5 & 8 \\
\hline
\end{tabular}

Tabla 1 Distorsión armónica máxima permitida Fuente: Especificación CFE-L000045

Tomando la formula (2) para verificar el porcentaje de la distorsión de armónica total de corriente $T H D$ en la fase $\mathrm{AN}$, se tomaros los datos del histograma del 10/07/19 15:05 h, al 11/107/19 15:05 h, los datos se muestra en la tabla 2, del $I h_{3}$ al $I h_{17}$, tomando solo los armónicos impares. El valor de la corriente fundamental se tomó el promedio de la fase A, en la mismas fecha como se muestra en la figura 13 dando una corriente de 110 Amperes.

\begin{tabular}{|l|r|}
\multicolumn{1}{c}{$\begin{array}{c}\text { Corriente } \\
\text { armónico impar }\end{array}$} \\
\hline Ih3 & 20.55 \\
\hline Ih5 & 6.61 \\
\hline Ih7 & 5.74 \\
\hline Ih9 & 2.41 \\
\hline Ih11 & 4.02 \\
\hline Ih13 & 4.44 \\
\hline Ih15 & 1.34 \\
\hline Ih17 & 2.26 \\
\hline
\end{tabular}

Tabla 2 Corrientes Armónicos en (\%) fase AN Fuente: Analizador de redes

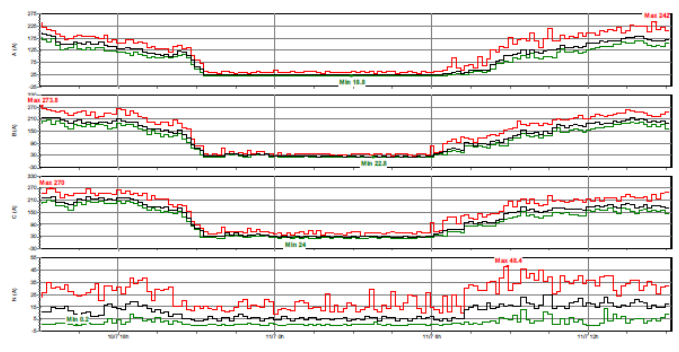

Figura 13 Corriente fundamental Fuente: Elaboración Propia

Sustituyendo los valores en la ecuación (2)

$T H D=\frac{\sqrt{548}}{110} * 100=21 \%$

Coincidiendo con el valor de THD de corriente de la figura 12 .
Para determinar el valor de Distorción armónica total de demanda (DATD) en la tabla 2 , la tolerancia es menor de $8 \%$, con los valores de la corrientes armónicas se sustituye en la ecuación 3. La Corriente Máxima de Carga IL, correspondiente al promedio de las corrientes de demanda máxima de los últimos 12 meses.

Para la fase A:

$T D D=\frac{\sqrt{548}}{243} \times 100=10 \%$

Se encuentra arriba del nivel permitido en las normas IEEE Std 519-2014 y la Especificación CFE L0000-45.

Durante las mediciones el día 10/07/19 a las 14:05:30, en la figura 14 se muestran los armónicos de corriente en el neutro en opción histograma en gráfico de barras, se muestra el porcentaje de contribución de cada uno de los componentes relacionados con la señal total, el tercer armónico indica el $100 \%$, y en el encabezado de la pantalla indica una distorsión armónica total (THD) de $183 \%$ y un factor K de 8.7

El factor K, se mide para la corriente y la potencia, este número que cuantifica la sobrecarga potencial en transformadores debida a corrientes de armónicos. Los armónicos de orden superior influyen en el factor K más que los armónicos de orden bajo. [1].

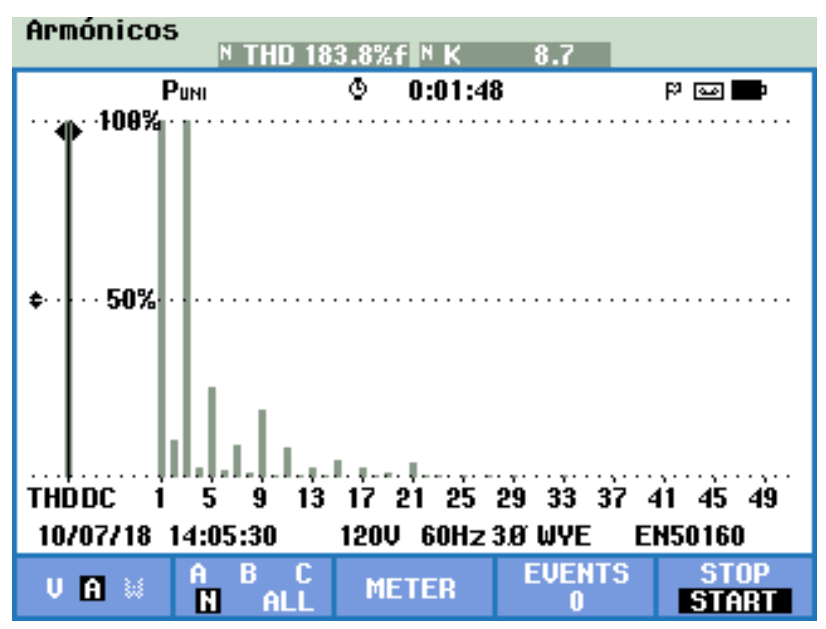

Figura 14 Gráfico de barra de corrientes del neutro en (\%) y su contribución (THD)

Fuente: Elaboración Propia 


\section{Frecuencia/Desequilibrio}

Frecuencia.- El equipo analizador proporciona información referente a Frecuencia /desequilibrio, se observa en la figura $15 \mathrm{de}$ variaciones de frecuencia, que se tienen valores máximo, media y mínimo, el día 11/07/2019 a las 18:08 h, se tiene un valor de mínimo de $59.903 \mathrm{~Hz}$, sobrepasando el límte establecido en [6], donde la frecuencia de $60 \mathrm{~Hz}$ tiene una tolerancia de $0.8 \%$ en más o menos, y el día $15 / 07 / 2019$ a las $4: 45 \mathrm{hr}$ se tiene un valor máximo de 60.088 estando dentro del porcentaje permitido, en forma general en los valores medio de variaciones de frecuencia se tiene estabilidad durante el tiempo de medición.

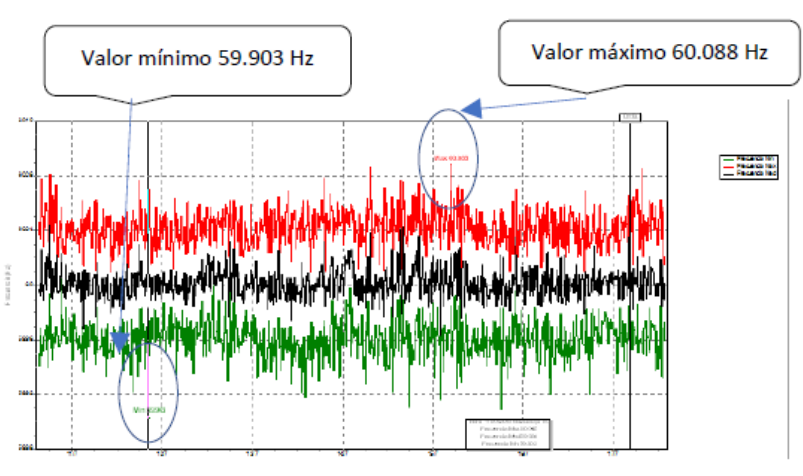

Figura 15 Variación de frecuencia

Fuente: Elaboración Propia

Desequilibrio de tensión.-

Desequilibrio de voltaje negativo.- (Unbal Vn\%) Se observa en la figura 16 el día 10/07/19 a las 9:55h se tiene un valor de desequilibrio maximo de $7.9 \%$, según [1] el desequilibrio de tensión negativa no debe sobrepasar el 2\%. En los dias posteriores de la medición se tienen registrados eventos que no sobrepasa el $2 \%$ indicado.

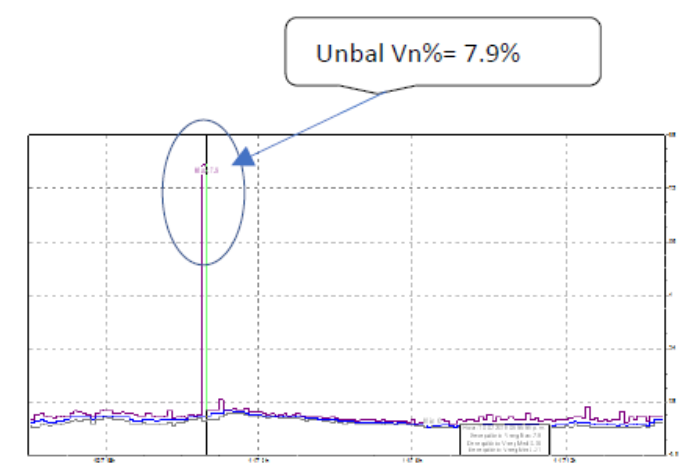

Figura 16 Desequilibrio de tensión Fuente: Elaboración Propia
Lo deseable para los parametros regulados conforme al Reglamento de la Ley de Servicio Público de Energía Eléctrica, es que mantengan un comportamiento estable en el alcance nominal, dentro de rangos normalizados y con forma de onda sinusoidal perfecta; sin embargo en el desempeño operativo de las instalaciones tanto las del suminstrador como las del cliente se tienen factores, perturbaciones, que afectan a los parámetros mencionados, la utilización misma de la energía suministrada es uno de los factores que causan la variación de dichos parametros.

\section{Huecos y picos}

Los huecos y picos se muestran en una tabla de clasificación de graficos de CBEMA (Computer Business Equipment Manufacturers Association) linea azul, e ITIC (Information Technology Indutry Council) línea roja, según EN 50160.

Están trazados los marcadores de curva de cada hueco y pico. La altura del eje vertical muestra la gravedad del hueco o del pico en relación con la tensión nominal. La posición horizontal muestra la duración del hueco o del pico, Estas curvas muestran una envoltura de tensión de entrada de corriente alterna que normalmente se puede tolerar (sin interrupción de la función) por la mayoría de equipos informaticos (ITE).[1].

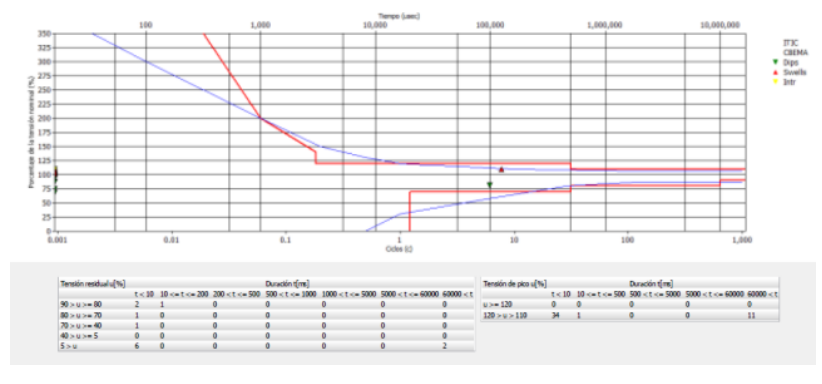

Figura 17 Huecos y picos

Fuente: Elaboración Propia

En la figura 17 se observa el comportamiento de la tensión nominal dado en porcentaje (valor nominal al 100\%). Por lo que lo ideal es que la tensión se mantenga de manera constante a través del tiempo en un valor del $100 \%$. Cuando se tiene alguna subida o bajada de tensión, el valor se incrementará o disminuirá respectivamente en proporción al valor nominal. En esta figura se aprecia el aumento de las tensiones por medio de las viñetas de colores, estas tensiones, corresponden con los valores de fase a neutro de las fase A, B y C.

MARTÍNEZ-RODRÍGUEZ, Guillermo Miguel, CRUZ-ISIDRO, Berenice de Jesús, GARRIDO-MELENDEZ, Javier y JIMÉNEZRIVERA, Jesús. Análisis de la calidad de energía eléctrica en una subestación de 300 Kva. Revista de Operaciones Tecnológicas. 2019 
En 1 aparte inferior de las curvas, se muestra una tabla Tensión residual u [\%] (caídas de tensión) y otra de Tensión de pico u[\%] (aumentos de tensión). Estas tablas muestran el número de veces que se tuvieron estos fenómenos dados en porcentaje del valor nominal y su duración. En la tabla de Tensión residual u[\%], en el primer renglón, indica que se presentaron 3 caidas de tensión en una proporción del $80 \%$ a $90 \%$ de la tensión nominal, 2 con una duración menor a $10 \mathrm{mseg}$ y 1 con una duración mayor a $10 \mathrm{mseg}$ y menor a $200 \mathrm{mseg}$.

\section{Recomendaciones}

Continuar con el análisis de la calidad de energía eléctrica en las cargas eléctricas secundarias para determinar que equipos son los que ocasionan distorsión en la señal eléctrica, así como determinar cuál es el motivo de estas desviaciones.

\section{Resultados}

La interpretación de los parámetros eléctricos muestra que algunos están fuera de los límites permitidos, como es el incremento de armónicos de corriente en el neutro, se analizan los eventos de ondas, parpadeos, etc.

\section{Agradecimiento}

Se agradece a la Universidad Veracruzana campus Coatzacoalcos que permitió sus instalaciones y el equipo necesario para la realización de este estudio, y a los alumnos y docentes que participaron en su elaboración y comentarios.

\section{Conclusión}

El propósito del estudio de la calidad de energía es que los alumnos realicen las conexiones eléctricas en el lado de baja tensión en un transformador energizado, conservando las medidas de seguridad establecidas, así como obtener información de los parámetros eléctricos, y analizar si se encuentran dentro de lo que indican las normas, y así determinar cuáles pueden afectar el buen desempeño de los propios equipos o afectar de manera indirecta a los usuarios de ellos, también sirve de inicio para tomar medidas y continuar con el estudio para su corrección.

\section{Referencias}

[1] Fluke 434-II, (2012). Manual del fabricante Analizador trifásico de energía y calidad de la energía.

[2] Carpio, I. J., Míguez, C. J. V., \& Guirado, T. R. (2013). Alimentación de cargas críticas y calidad de la energía eléctrica. Retrieved from https://ebookcentral.proquest.com

[3] Especificación CFE L0000-45 (2005) "Desviaciones permisibles en las formas de onda de tensión y corriente en el suministro y consumo de energía eléctrica".

[4] IEEE Std 1159-1995 Recommended Practice for Monitoring Electric Power Quality.

[5] GUÍA CFE L0000-70 (2009) “Calidad de la energía: Características y límites de las perturbaciones de los parámetros de la energía eléctrica".

[6] Reglamento de la ley del servicio público de energía. Publicado DOF 30-11-2012.

[7] Lucas Gómez Lizzie (2019). "Estudio de la calidad de la energía en la subestación eléctrica de 300 kVA de la Universidad Veracruzana campus Coatzacoalcos". Universidad Veracruzana, Coatzacoalcos, México.

[8] IEEE. (2014). IEEE Std 519-2014 (Revision of IEEE Std 519-1992) - Redline: IEEE Recommended Practice and Requirements for Harmonic Control in Electric Power Systems Redline. IEEE

[9] IEEE Std 399-1997 IEEE Recommended Practice for Industrial and Commercial Power Systems Analysis

[10] IEEE Std 519 ${ }^{\mathrm{TM}}-2014$ (Revision of IEEE Std 519-1992) IEEE Recommended Practice and Requirements for Harmonic Control in Electric Power Systems.

[11] Carpio, I. J., Míguez, C. J. V., \& Guirado, T. R. (2013). Alimentación de cargas críticas y calidad de la energía eléctrica. Retrieved from https://ebookcentral.proquest.com.ezproxy.cdig ital.uv.mx:8443.

[12] Dsib Jiménez Sergio. (2017). Simulación de Armónicos en Matlab. Revista de Análisis Cuantitativo y Estadístico. in Volume 4 Number 10, with ISSN: $2410-3438$, at pages $15-25$ segment.

MARTÍNEZ-RODRÍGUEZ, Guillermo Miguel, CRUZ-ISIDRO, Berenice de Jesús, GARRIDO-MELENDEZ, Javier y JIMÉNEZRIVERA, Jesús. Análisis de la calidad de energía eléctrica en una subestación de 300 Kva. Revista de Operaciones Tecnológicas. 2019 